chemistry, concerned not with the outer layers of the atom but with the nucleus itself. The transmutations of one element into another involves adding or subtracting a particle, charged or uncharged, to or from the nucleus, and this may be effected in many cases by bombardment with foreign particles. A few of these particles may enter the nucleus, and this may sometimes lead to the emission of a particle from the nucleus itself. The first of such transmutations was accomplished in 1919, when nitrogen was disintegrated by $\alpha$-particle bombardment with the liberation of fast protons. More recently, a new type of disintegration has been discovered in which a neutron is emitted. In these cases the residual nucleus in the transformations is stable. In the cases investigated by $\mathrm{M}$. and Mme. Curie-Joliot, an artificial radioactive element is formed by bombarding a light element with $\alpha$ particles. Fermi and his collaborators have found that a very large number of elements can be disintegrated by neutron bombardment, giving artificial radioactive elements. The neutron, on account of its lack of charge, can penetrate the heavy nuclei when $\alpha$-particles would be turned back. Finally, Lord Rutherford directed attention to the accomplished production of nuclear disintegration, using bombarding particles artificially accelerated by high voltages instead of the particles emitted from natural radio-elements.

\section{Science and Road Traffic}

In his lecture before the British Science Guild on December 19, Col. Mervyn O'Gorman discussed the application of science to the problems of road traffic. Road traffic, he said, is not replaceable by other distributive agencies, and its prosperity is indeed advantageous to them. The magnitude of motor transport as an industry is such that it has more employees, involves more capital wealth, and pays larger taxation to the State than almost any other industry in England. Inprovement which is being, and must be, sought in safety of distribution by road involves getting the largest amount of road distribution achieved per single accident. The business of evolving the necessary instruments, the analytical methods, the interpretation of data, and similar work on the accident ratio is the proper function of science, especially physics, mechanics, mathematies, chemistry, geology, metallurgy, statistics, physiology, psychology, etc. A committee should be formed to advise and undertake research, and it should not contain road interests (financial or professional) and it should receive all the specific 'road and traffic' information that it needs from the Ministries of Transport and of Health, the Home Office and from witnesses. Following the precedent of the successful Aeronautical Research Committee at its foundation in 1908 , it should report direct to the First Lord of the Treasury. Its members should be paid, and should all be scientific men, preferably nominated by the Royal Society in conjunction with the Department of Scientific and Industrial Research. The committee having been formed, it should be free to formulate and verify its own theories in the study of safe traffic flow, economic flow, pedestrian flow, etc., these being the frameworks of various long-range researches.

\section{Maternal Mortality}

SIR Huton Young, the Minister of Health, received on December 11 a deputation from the Maternal Mortality Committee. Mrs. H. J. Tennant, introducing the deputation, said that it represented more than 3,000,000 women and was the outcome of a meeting on the subject of maternal mortality held in November. Mrs. Barton said that malnutrition, though not a primary cause, is a contributing factor to maternal mortality, and she fears that the block grant system of Exchequer grants is less effective in stimulating local authorities than the former percentage grants. Lady Barrett dealt with the question of ante-natal care, and the necessity of improving the training of doctors and midwives. Miss Gregory considers that midwives ought to have a two- or three-year course in a first-class hospital. Other speakers stressed the importance of maternity and child welfare services. The Minister, in reply, said that the problem of maternal mortality is giving him grave concern. The maternity and child welfare services of local authorities are being steadily developed, and he considers that no financial check has been placed upon them by the alteration in the grant system. There is no evidence that there is any close relation between malnutrition and a high maternal mortality rate. Nevertheless, the conditions in depressed areas are such as to give rise to anxiety, and the position is receiving the close attention of the administration. $\mathrm{He}$ outlined measures that are being taken to improve maternity and child welfare services throughout Great Britain, and alluded to special inquiries and investigations that are being made in districts where the maternal mortality rate is abnormally high.

\section{Further Tests of the Medium Rudi Schneider}

IN the Proceedings of the Society for Psychical Research for October is published a further report on the alleged psychic phenomena occurring in the presence of the medium Rudi Schneider. Under the joint authorship of Mr. T. Besterman and Mr. O. Gatty, the paper describes an attempt to look for confirmation of the infra-red phenomena previously reported, and generally to conduct tests by instrumental means. As an example of the kind of methods to be used in experimental work with the so-called physical phenomena, the report seems to be a step in advance, and the results suggest that through such instrumental means a better idea of the nature of the phenomena may be obtained. Generally speaking, the present results were negative. The interruption of the infra-red rays as previously reported by Dr. Osty in Paris and by others in Great Britain received no confirmation, in spite of a series of careful observations; and through the help of of Dr. C. G. Douglas it was ascertained that the medium's breathing, which was considered of sufficient interest to reproduce in a recent series of talks 